\title{
AUV Link Mobile Ad-Hoc Network Examination
}

\author{
Igor S. Konstantinov, G. S. Vasyliev, Oleg R. Kuzichkin, D. I. Surzhik, I. A. Kurilov, \\ Sergey A. Lazarev
}

\begin{abstract}
Wireless optical communication systems allow reliable communication in the ultraviolet $(U V)$ range, and protected from intentional suppression and interception, both in the presence and in the absence of direct visibility between the transmitter and the receiver. Currently, the actual and insufficiently explored direction is the development and research of mobile ad-hoc networks (MANET) with a UV communication channel. To analyze the capacity of a mobile network of $U V$ communications with different spatial orientation of network nodes, it is necessary to take into account not only their coordinates and elevation angles but also azimuths. NLOS UV bandwidth models have been developed using three types of modulation: on-off keying (OOK), pulse-phase modulation (PPM), digital pulse interval modulation (DPIM) with a different number of guard intervals. The noted advantages of DPIM, which are most important for the implementation of a mobile self-organizing network with a UV channel, are: it does not require symbolic synchronization of the transmitter and receiver, it requires a smaller frequency band compared to PPM, it has a higher energy efficiency compared to OOK. A parametric approximation of losses in the NLOS UV channel has been performed, taking into account the azimuthal deviations of the transmitter and receiver for specific parameters of the communication system. Has been done a performance simulation of the MANET network with an ultraviolet communication channel Has been calculated values of the bit error rate (BER), and bit rate for a system with different types of modulation with low, medium, and high noise levels in the channel. With the use of parametric approximation of losses in the channel, are analyzed the BER and the bitrate of the $U V$ communication system with an azimuthal deviation within 30 degrees. Based on the obtained simulation results, prospects for the further development of mobile self-organizing networks with a UV channel are indicated to improve their reliability and performance.
\end{abstract}

Keywords : wireless ultraviolet communication; Mobile Ad-Hoc Network, MANET; OOK; PPM; DPIM; bit error rate, BER.

Revised Manuscript Received on July 22, 2019

Igor S. Konstantinov, Belgorod State University, 85 Pobedy str., Belgorod, Russia

G. S. Vasyliev, Belgorod State University, 85 Pobedy str., Belgorod, Russia

Oleg R. Kuzichkin, Belgorod State University, 85 Pobedy str., Belgorod, Russia

D. I. Surzhik, Belgorod State University, 85 Pobedy str., Belgorod, Russia

I. A. Kurilov, Belgorod State University, 85 Pobedy str., Belgorod, Russia Sergey A. Lazarev, Belgorod State University, 85 Pobedy str., Belgorod, Russia

\section{INTRODUCTION}

Ultraviolet (UV) wireless optical communication systems provide reliable communication protected from intentional suppression and interception, both in the presence and in the absence of direct visibility between the transmitter and receiver (non-line-of-sight mode, NLOS). A significant number (more than a hundred) of scientific articles, in particular, [1-6], are devoted to the problems of modeling and experimental testing of UV communication systems, especially the "sun-blind" UV-C radiation range with a wavelength of no more than $280 \mathrm{~nm}$.

Currently, the actual and insufficiently explored direction is the development and research of mobile ad-hoc networks with a UV communication channel (MANET). The MANET concept $[7,8]$ can be used to create a secure, wireless, self-organizing, special-purpose network capable of operating at important and dangerous facilities (when conducting search and rescue operations, preventing and eliminating emergency situations, working under the conditions of the impact of destructive and damaging factors of natural and man-made law enforcement operations, counter-terrorism operations, tactical military operations). The use of UV radiation in MANET both as the main communication method and in combination with radio frequency will increase the reliability and information security of such networks.

To analyze the effectiveness of the MANET network with a UV channel, it is necessary to investigate its performance in various conditions. Such conditions are the noise level, the spatial location of network nodes, the energy characteristics of the transmitter and receiver. Performance characteristics of the communication system are the bit error rate (BER) and system bit rate. In addition, the analysis should determine the most promising types of modulation for MANET networks with a UV channel.

The main aim of the work is to simulate and analyze the performance characteristics of a mobile wireless ad-hoc network with an ultraviolet communication channel using various modulation methods.

\section{GEOMETRY OF UV NLOS CHANNEL}

The standard geometry of the UV channel model in the non-line-of-sight mode (NLOS UV) used in a large number of works, for example, [9-11], is shown in Figure 1a. The figure shows the vertical projection of

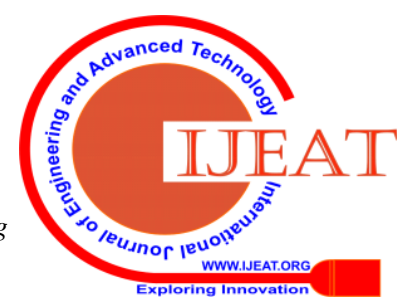




\section{AUV Link Mobile Ad-Hoc Network Examination}

the channel and is designated: $T x$ is the transmitter, $\mathrm{Rx}$ is the receiver, $r$ is the distance between $T x$ and $R x, \theta 1,2$ and $\varphi 1,2$ is the elevation angle and width of the DP, index 1 refers to the transmitter, index 2 to the receiver, $\theta$ s is the scattering angle, $\mathrm{V}$ is the total volume of $\mathrm{Tx}$ and $\mathrm{Rx}$ radiation patterns, $\mathrm{r} 1,2$ is the distance from $\mathrm{Tx}$ and $\mathrm{Rx}$ to the center of region $\mathrm{V}$. To analyze the bandwidth of a mobile UV network with different spatial orientation of network nodes, it is also necessary to take into account the azimuths of the transmitter and receiver $\psi \mathrm{T}, \mathrm{R}$, as shown in Figure $1 \mathrm{~b}$.

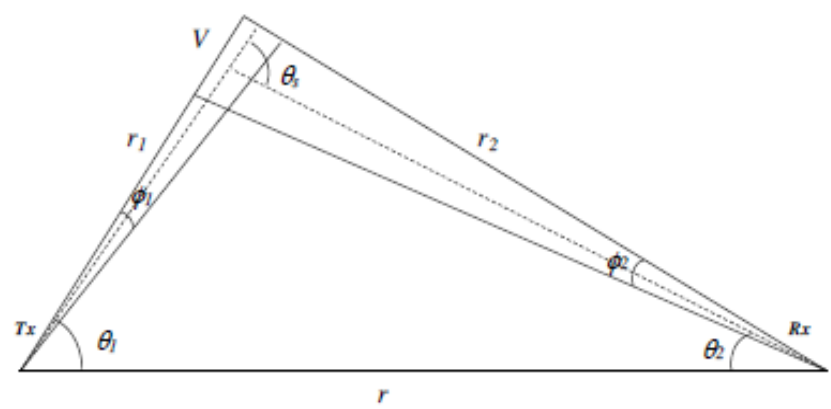

(a)

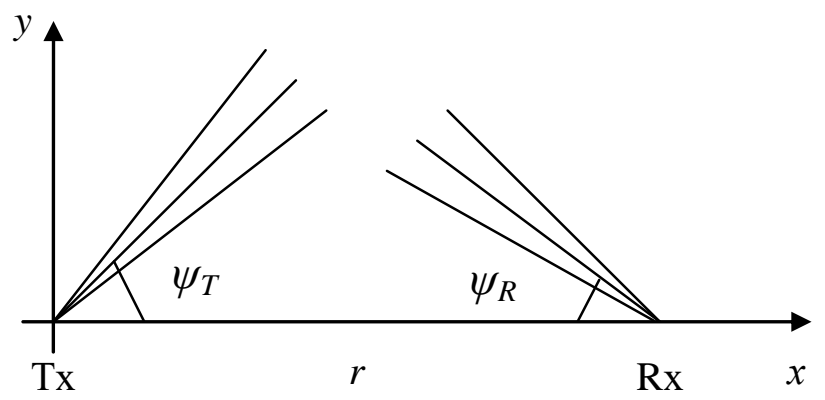

(b)

Figure 1 - Vertical (a) and horizontal (b) projection of the UV channel in the of non-line-of-sight mode (NLOS UV)

\section{UV NLOS CHANNEL BANDWIDTH MODELS USING VARIOUS MODULATION TYPES}

According to $[10,12]$, with ideal detection of on-off keying modulation $(\mathrm{OOK})$, the probability of bit errors (BER) is determined by the expression:

$B E R=0.5 \exp \left(-\frac{P_{t} \eta_{f} \eta_{r} \lambda}{\operatorname{Loss} \cdot R h c}\right)$

where $P_{t}$ is the transmitter radiation power, $\eta_{f}$ is the filter transmission coefficient, $\eta_{r}$ is the detector (receiver) quantum efficiency, $\lambda$ is the wavelength, Loss is the channel loss, $R$ is the bitrate, $h=6,626 \cdot 10^{-34} \mathrm{~J} \cdot \mathrm{s}$ is the Planck constant, $c=3 \cdot 10^{8}$ mps is the speed of light in vacuum.

The disadvantage of the model (1) is that it does not take into account the effect of noise. The article [13] contains generalized expressions for the dependence of BER on the signal-to-noise ratio (SNR) for an abstract communication channel, but does not take into account the specifics of the UV channel:

$$
\begin{aligned}
& B E R_{\mathrm{OOK}}^{\mathrm{NRZ}}(S N R)=\frac{1}{2} \operatorname{erfc}\left(\frac{1}{2 \sqrt{2}} \sqrt{S N R}\right) \\
& B E R_{\mathrm{OOK}}^{\mathrm{RZ}}(S N R)=\frac{1}{2} \operatorname{erfc}\left(\frac{1}{2} \sqrt{S N R}\right) \\
& B E R_{\mathrm{L}-\mathrm{PPM}}(S N R)=\frac{1}{2} \operatorname{erfc}\left(\frac{1}{2 \sqrt{2}} \sqrt{S N R \cdot \frac{L}{2} \log _{2} L}\right)
\end{aligned}
$$

where NRZ is coding without returning to zero, RZ is coding with returning to zero, L-PPM is L-positional pulse-phase modulation, $\quad L=2, \quad 4, \quad 8, \quad 16, \quad \ldots$; $\operatorname{erfc}(x)=\frac{2}{\sqrt{\pi}} \int_{x}^{\infty} \exp \left(-t^{2}\right) d t$ - error function (probability integral).

To take into account the UV channel specific features, it is necessary to calculate the SNR as the ratio of the detected signal photons number $N_{d}$ to the number of noise photons $\quad N_{n} \quad$ as $\quad S N R=N_{d} / N_{n}$, where $N_{d}=\eta_{f} \eta_{r} N_{r}=\eta_{f} \eta_{r} N_{r} /$ Loss $=\eta_{f} \eta_{r} P_{t} \lambda /($ hcR $\cdot$ Loss $)$

. The measurement results showed that typical detection frequencies of noise photons by a receiver with an aperture of $A_{r}=1,77 \mathrm{~cm}^{2}$ with low, medium and high noise levels are about 1000; 5000; 15,000 Hz, respectively [14].

It should be noted that OOK modulation is used most frequently in UV communication systems since its technical implementation is simpler compared to PPM modulation. At the same time, PPM modulation has higher energy efficiency but based on the symbol synchronization of the transmitter and receiver. Digital pulse interval modulation (DPIM) can modulate the same number of bits per symbol as an even-order PPM but does not require symbol synchronization. It is also important that DPIM uses fewer time slots per symbol than PPM and thus requires less bandwidth [15-20]. As PPM, DPIM has higher energy efficiency than OOK. These advantages determine the relevance of using DPIM in various UV communication systems.

The noted features are especially significant for the implementation of the MANET network with a UV channel since a large number of network subscribers in a limited space use a significant frequency resource. The narrow range of UV LEDs available on the market, especially in the most promising for the sun-blind UV-C communication, makes it extremely difficult to organize multiple access with wavelength separation. Modulation of the frequency and other parameters of the emitted signal directly in the UV range require the bulky optical filters use, which unacceptably deteriorate the device's mass-size critical parameters for the mobile system. In addition, approaches and solutions for the synchronization of the UV radiation transmitter and the receiver based on the use of an external clock signal, in contrast to the radio frequency range, have not been developed. Therefore

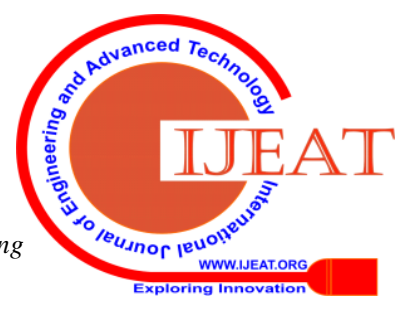


the use of self-synchronizing modulation and coding methods, economically using the available frequency band, and DPIM in particular, for mobile UV networks is highly

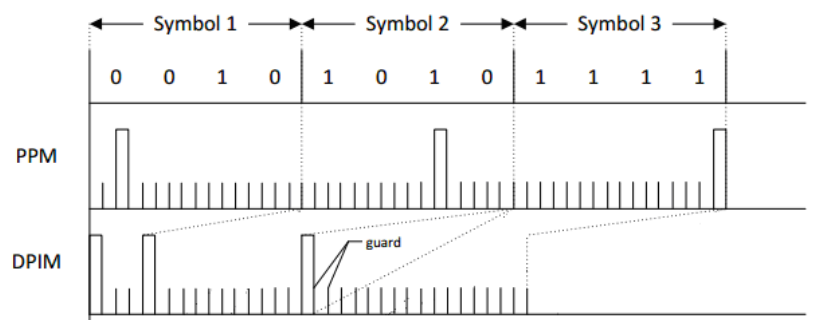

Figure 2 - PPM and DPIM modulation timing diagrams, $\mathrm{M}=4$, the number of guard intervals $\mathrm{n}=1$

The same expression determines the average number of signal photons accumulated during the symbol interval for PPM and DPIM [15]

$$
\lambda_{s}^{P P M}=\lambda_{s}^{D P I M}=\frac{P_{t} \eta_{f} \eta_{r}}{\frac{R}{M} \operatorname{Loss} \cdot h v},
$$

where $v$ is the radiation.

Following expressions determine the number of noise photons accumulated during the symbol interval for PPM and DPIM:

$$
\lambda_{b}^{P P M}=\frac{N n}{\frac{R}{M} \frac{2^{M-1}+2 n+1}{2}}, \quad \lambda_{b}^{D P I M}=\frac{N n}{\frac{R}{M} 2^{M}}
$$

Based on the comparison of expressions (5) and (6), the ratio of SNR parameter values for DPIM and PPM is:

$$
\frac{S N R^{D P I M}}{S N R^{P P M}}=\frac{2^{M-1}+2 n+1}{2 \cdot 2^{M}} .
$$

Next, obtain the BER dependence for DPIM from formulas (4) and (7) and taking into account $L=2^{M}$ :

$$
B E R_{\mathrm{DPIM}}(S N R)=\frac{1}{2} \operatorname{erfc}\left(\frac{1}{2 \sqrt{2}} \sqrt{S N R \cdot \frac{L}{2} \frac{L+2 n+1}{2 L} \log _{2} L}\right)
$$

\section{PARAMETRIC APPROXIMATION OF THE NLOS UV CHANNEL LOSSES, WITH CONSIDERING AZIMUTHAL DEVIATION OF THE TRANSMITTER AND RECEIVER}

The magnitude of the losses, which determines the performance of the UV communication system, is determined by the channel parameters: the communication range, the elevation angles and the azimuths of the transmitter and receiver, and the widths of their radiation patterns. The following parametric relation [14] describes desirable. Figure 2 shows the time diagrams of the 4-position pulse modulations PPM and DPIM with the number of guard intervals $\mathrm{n}=1$.

the dependence of losses in the UV channel on the distance r:

$$
\operatorname{Loss}(r)=\xi r^{\alpha} e^{\beta r},
$$

where - the parameters of a particular channel. For small communication distances (the first hundreds of meters), the effect of the exponential factor is insignificant, and the simplified model is valid:

$$
\operatorname{Loss}(r)=\xi r^{\alpha} \text {. }
$$

A specific feature oф UV communications mobile networks is the frequent movement of subscribers, which makes it difficult to ensure that the azimuths of the transmitter and receiver are pointing at each other (for example, by the maximum signal). At the same time, the NLOS mode remains relevant if there are obstacles in the communication distance (buildings, structures, or natural uneven terrain). The results of modeling and experimental studies of the UV channel with varying azimuths of the source and detector in the literature are quite rare [21]. In article [22] authors obtained the calculated loss dependence for various azimuths.

The following parabolic expression, based on the simulation results, describes the loss of the azimuthal deviation Tx and Rx $\Delta \psi$ (in degrees) with good accuracy for practice (error less than $3 \mathrm{~dB}$ ):

$$
\operatorname{Loss}(\Delta \psi)=L_{0}+K \cdot \Delta \psi^{2} \mathrm{~dB},
$$

model parameters: L0 - constant term (the channel losses value in the absence of azimuthal deviations); $\mathrm{K}$ - scale factor. The model is valid in the range of absolute values $\Delta \psi$ from 0 to 35-400, with further growth, $\Delta \psi$ losses remain approximately constant. The model parameters for a specific case calculated in [22]: the constant term $\mathrm{LO}=102 \mathrm{~dB}-$ is the channel losses in the absence of azimuthal deviation; $\mathrm{K}=0.22$ - scale factor. The model determines the increase in losses from the lowest value of 102 дБ to $137.2 \mathrm{~dB}$ at $\Delta \psi=400$, which is in good agreement with the simulation results for different azimuths in the paper [21] at Fig. 5b. Were taken the following values of the UV channel parameters: communication range $\mathrm{r}=100 \mathrm{~m}$, elevation angles of the transmitter and receiver $\theta 1=300$ and $\theta 2=500$, widths of the DP transmitter and receiver $\varphi 1=170$ and $\varphi 2=300$, emission wavelength $\lambda=260 \mathrm{~nm}$, scattering and absorption coefficients for clear weather, receiver aperture area $\mathrm{Ar}=1,77 \mathrm{~cm}^{2}$.

\section{RESULTS OF MODELING}

Based on the experimental frequency data, an equivalent number of noise photons were calculated during the pulse, determined by the bit rate and the specific modulation type. The SNR values are calculated, based on the obtained values; The corresponding dependences of BER on channel losses and on bitrate, calculated using expressions (2-4), are shown in Figures 3-6. The bitrate value was taken to be $R=1 \mathrm{Mbps}$ for calculating Figure 3 and 4 curves. The following communication system parameters were taken: transmitter radiation power $P_{t}$ 
$=50 \mathrm{~mW}$, filter transmission coefficient $\eta_{f}=0.1$, detector quantum efficiency $\eta_{r}=0.2$, wavelength $\lambda=260 \mathrm{~nm}$.

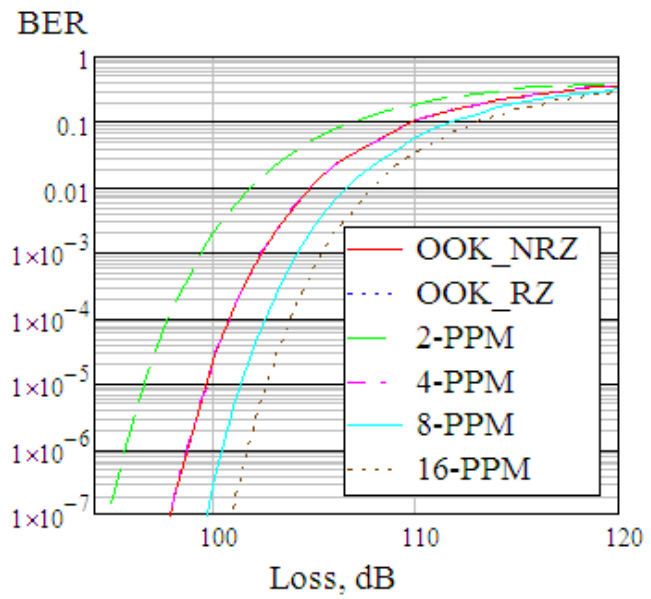

Figure 3 - Dependence of BER on channel losses for various types of modulation at a low noise level

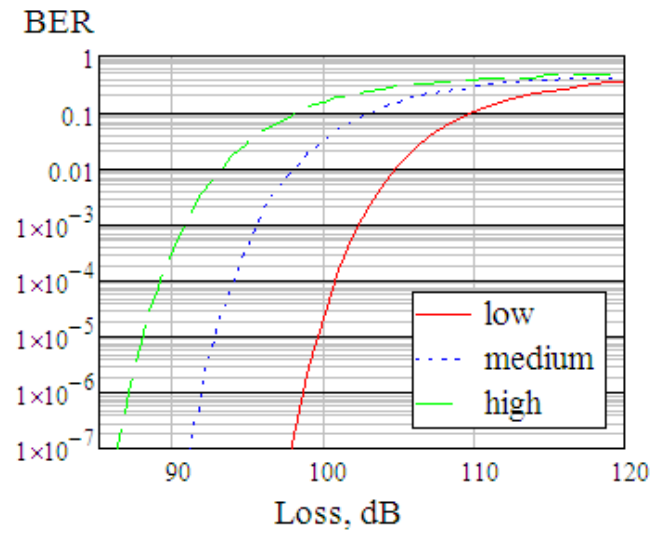

Figure 4 - Dependence of BER on losses for BER_NRZ modulation at various noise levels

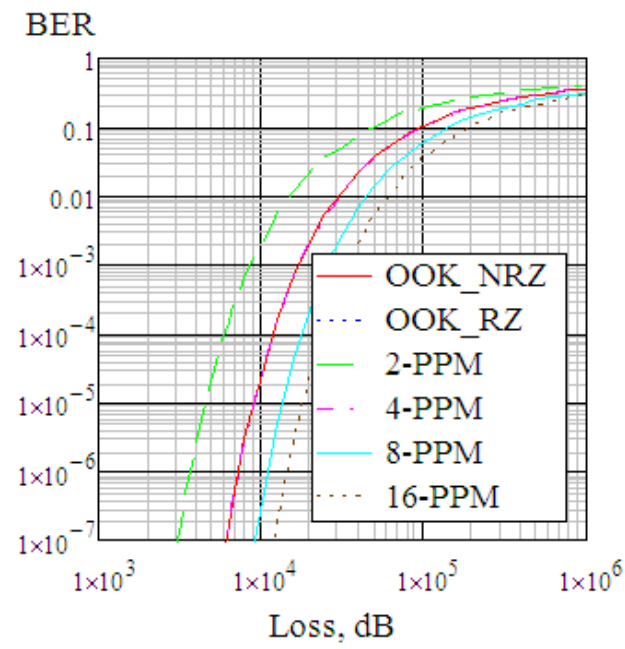

Figure 5 - Dependence of BER on the bitrate for various types of modulation at a low noise level

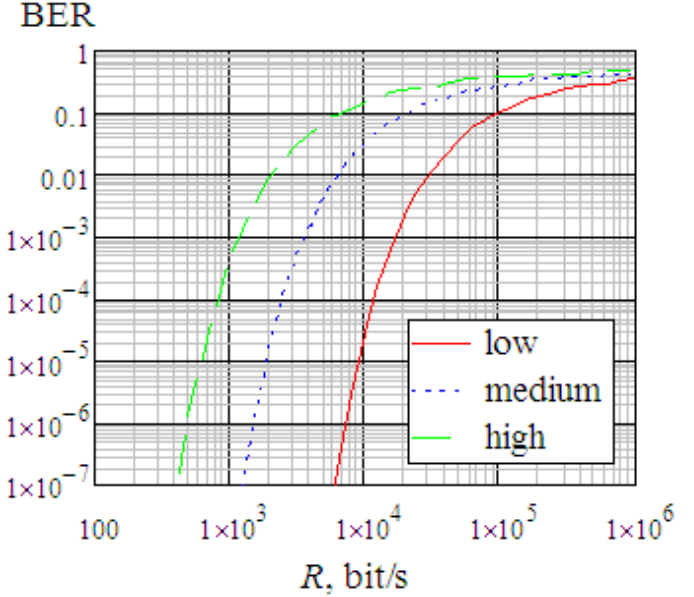

Figure 6 - Dependence of BER on the bitrate for BER_NRZ modulation at various noise levels

Dependencies showed a significant effect of noise on the bit error rate for various types of modulation. As can be seen from the last graph, keeping an acceptable value of $B E R=10^{-3}$ requires varying the bitrate from $17 \mathrm{kbps}$ with a low noise level, to 3,5 kbps with an average noise level, and up to 1,2 kbps with a large noise level.

The BER dependencies for PPM and DPIM, calculated using expressions (4) and (8), are presented in Figures 7-10. According to Figure 7, the difference in DPIM with $n=1$ and PPM is about $1.5 \mathrm{~dB}$ in favor of PPM. Additional protective intervals increase the noise immunity of DPIM, the DPIM gain in $\mathrm{dB}$ with the same BER value due to the introduction of each next guard interval after the first $(n=2,3,4)$ is about $0.7-1 \mathrm{~dB}$. The bitrate value was taken to be $\mathrm{R}=1 \mathrm{Mbps}$ for calculating Figure 7 and 8 curves.

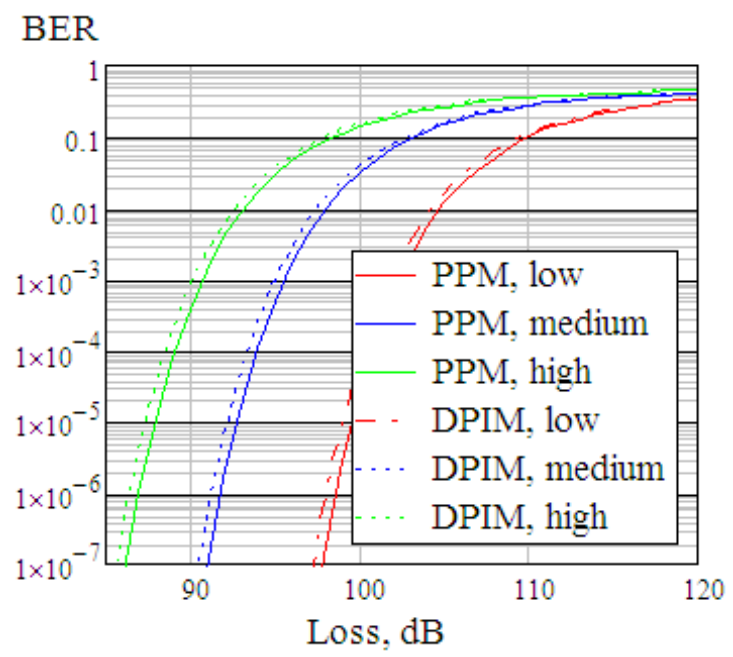

Figure 7 - BER loss dependencies for 4-PPM and 4-DPIM modulation with one guard interval $(n=1)$ at various noise levels 


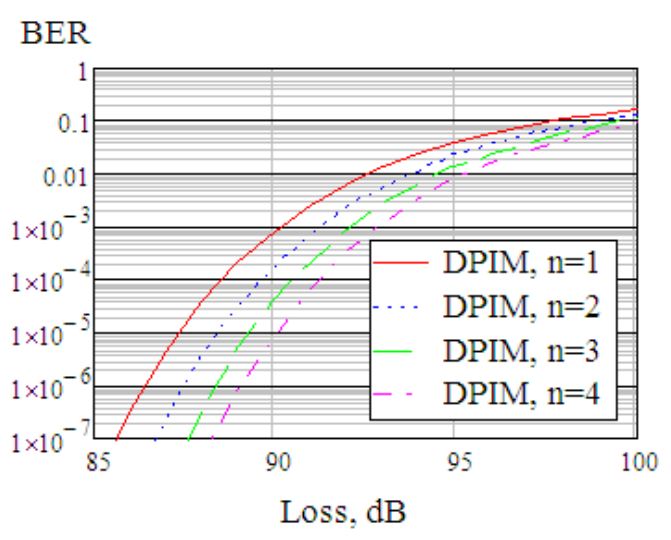

Figure 8 - Dependencies of BER on losses for modulation of 4-DPIM with different number of guard intervals $(n=1,2,3)$ at a high noise level

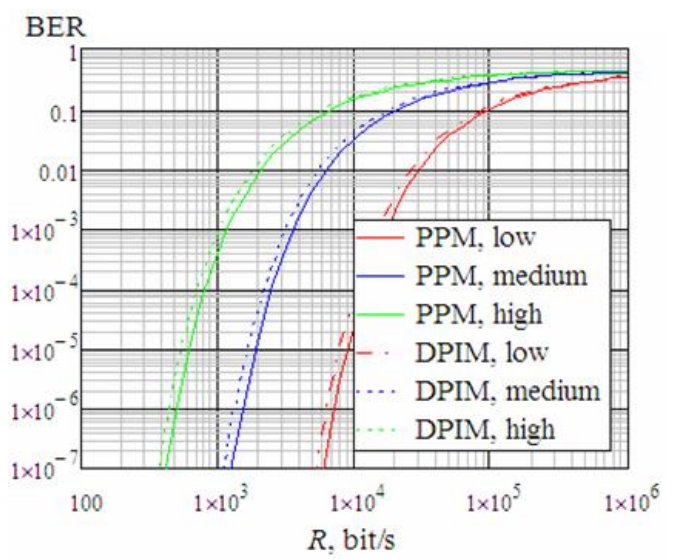

Figure 9 - Dependence of BER on the bitrate for 4-PPM and 4-DPIM modulation with one guard interval $(n=1)$ at various noise levels

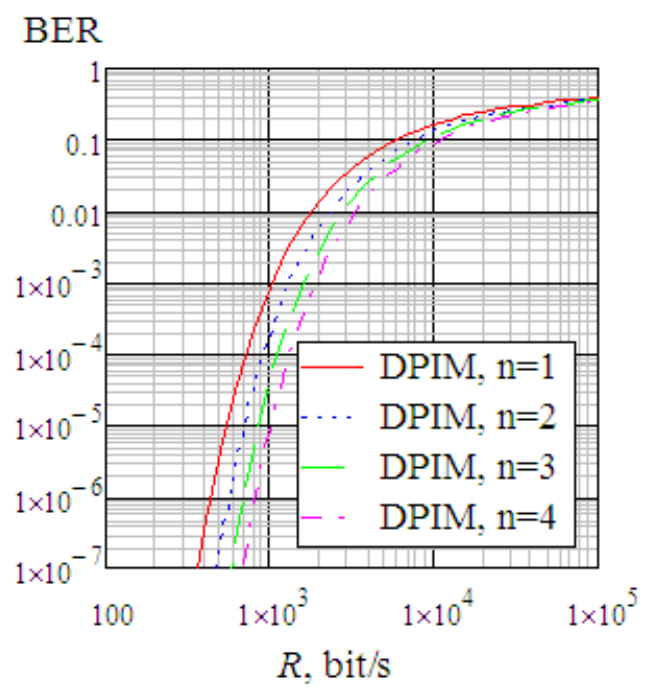

Figure 10 - Dependence of BER on the bitrate of 4-DPIM with different number of guard intervals $(\mathrm{n}=1,2,3)$ at a high noise level

Based on expressions (11) and (8), were calculated the dependences of BER on the azimuthal deviation $\Delta \psi$, shown in Figures 11-13. The bitrate is assumed to be $R=100 \mathrm{kbps}$.

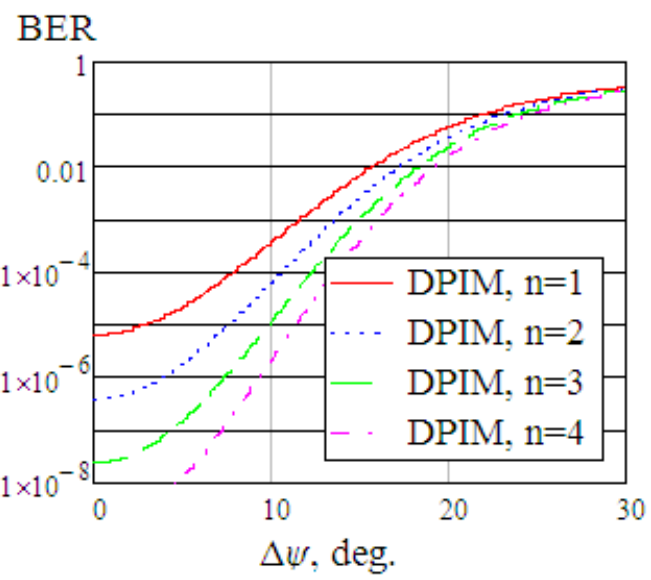

Figure 11 - BER dependence on azimuthal deviation $\Delta \psi$ for modulation of 4-DPIM with different number of guard intervals $(n=1,2,3,4)$ with an average noise level

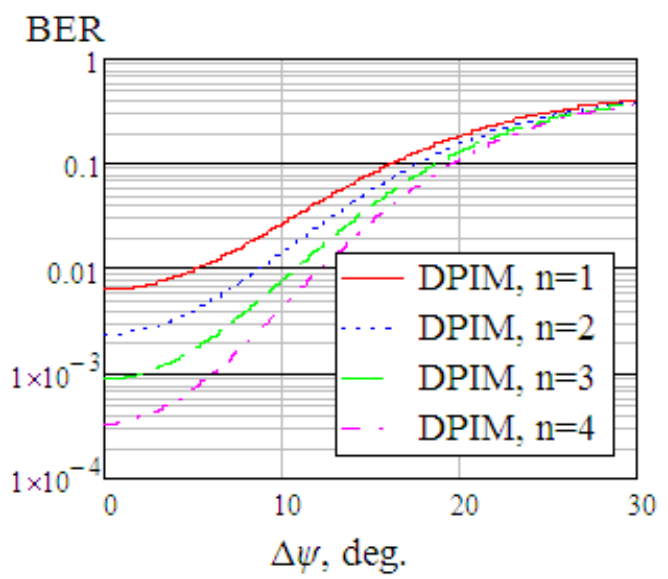

Figure 12 - BER dependence on azimuthal deviation $\Delta \psi$ for modulation of 4-DPIM with different number of guard intervals $(n=1,2,3,4)$ with a large noise level

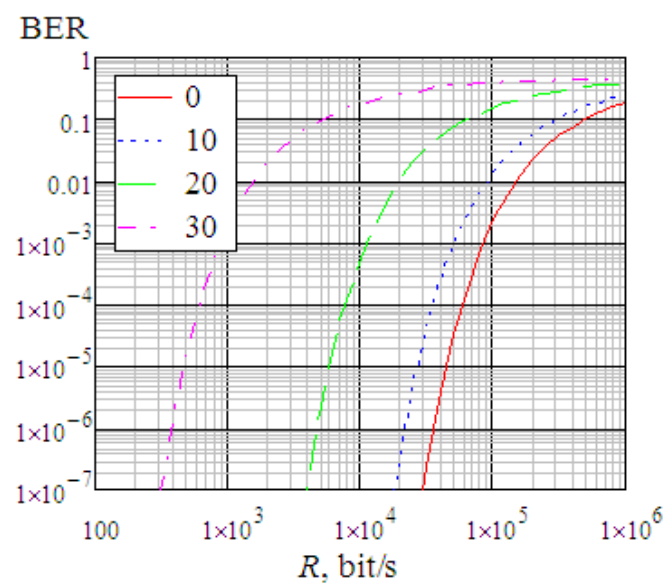

Figure 13 - Dependence of BER on the bitrate for modulation of 4-DPIM with two guard intervals $(n=2)$ with a large noise level, azimuthal deviation $\Delta \psi=0 ; 10 ; 20 ; 30$ degrees

The graphs show a strong dependence of BER on the noise level and azimuthal deviation. It follows from Figure 13 that with a strong influence of noise, to ensure an acceptable $\mathrm{BER}=10^{-3}$ due to imperfect

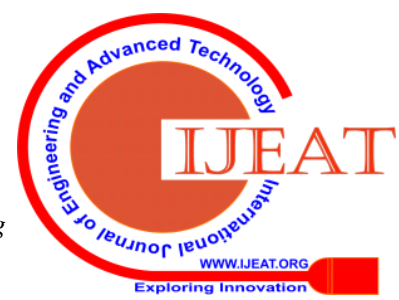




\section{AUV Link Mobile Ad-Hoc Network Examination}

pointing of the transmitter and receiver at each other, it is necessary to reduce the bitrate by two orders of magnitude (for $\Delta \psi=0^{0} R=85000 \mathrm{bps}$, for $\Delta \psi=30^{\circ} R=880 \mathrm{bps}$ ). in the range of azimuthal deviations of no more than $20^{\circ}$ achieved a reduction in the transmission rate within the same order: for $\Delta \psi=10^{0} R=51000 \mathrm{bps}$, for $\Delta \psi=20^{0} R=11000 \mathrm{bps}$.

\section{CONCLUSION}

In this article, has been simulated the performance of a mobile ad-hoc network with an ultraviolet communication channel. Also calculated the values of the bit error rate (BER) and bit rate at different channel noise levels for OOK, PPM, and DPIM modulation types. Are noted the advantages of DPIM, which are most important for the implementation of a mobile ad-hoc network with a UV channel: it does not require symbolic synchronization of the transmitter and receiver, it requires a smaller frequency band compared to PPM, it has a higher energy efficiency compared to OOK. Was performed a parametric approximation of the losses in the NLOS UV channel with considering the azimuthal deviation of the transmitter and receiver. On the basis of the performed approximation, were analyzed the BER and bitrate of the UV communication system at different azimuthal deviations. The analysis showed that a reduction in the transmission rate within the same order of magnitude with $\mathrm{BER}=10^{-3}$ is achieved in the range of azimuthal deviations of no more than $20^{\circ}$. Thus, to build transceiver modules of a mobile ad-hoc UV communication network, it is important to use antijamming coding, a data link layer protocol with automatic bitrate setting, an automatic guidance system for the transmitter and receiver orientation with an azimuth error of not more than $20^{\circ}$.

\section{ACKNOWLEDGMENTS}

This work was supported by the Ministry of Education and Science of the Russian Federation under Grant RFMEFI57518X0175 "Development of digital communication modules of mobile devices operating on the basis of UV data channels for establishing special purpose wireless self-organizing networks"

\section{REFERENCES}

[1] $\mathrm{Xu}$ Z. and Sadler B. Ultraviolet communications: potential and state-of-the-art IEEE Commun. Mag.4667-73, 2009.

[2] Elshimy M. and Hranilovic S. Non-line-of-sight single-scatter propagation model for noncoplanar geometriesJ. Opt. Soc. Am.A28420-8, 2011.

[3] Shaw G., Nischan M., Iyengar M., Kaushik S. and Griffin M. NLOS UV communication for distributed sensor systems Proc. SPIE412683-96, 2000 .

[4] Wang L., Li Y., Xu Z. and Sadler B. M. Wireless ultraviolet network models and performance in noncoplanar geometry. IEEE Globecom Workshopspp 1037-41, 2010.

[5] Wang L., Xu Z. and Sadler B. M. Non-line-of-sight ultraviolet link loss in noncoplanar geometry. Opt. Lett. 351263-5, 2010.

[6] Wang L., Xu Z. and Sadler B. M. An approximate closed-form link loss model for non-line-of-sight ultraviolet communication in noncoplanar geometry. Opt. Lett. 361224-6, 2011.

[7] Hekmat R. Ad-hoc networks: fundamental properties and network topologies. - Springer, 2006. - 154 p. ISBN-10 1-4020-5165-4 (HB).

[8] Polshykov K. A. Theoretical bases of packet data transmission in a wireless self-organizing network while providing communication at construction sites. - PhD thesis of speciality. 05.13.01 System analysis, management and information processing (construction and housing). - Belgorod, 2016.
[9] Luettgen M. R., Shapiro J. H. and Reilly D. M. Non-line-of-sight single-scatter propagation model. J. Opt. Soc. Am.A81964-72, 1991.

[10]Xu Z., H. Ding, B. M. Sadler, and Chen G., "Analytical performance study of solar blind non-line-of-sight ultraviolet short-range communication links" Optics Letters, vol. 33, no. 16, pp. 1860-1862, Aug. 2008.

[11]Hou W., Liu C., Lu F., Kang J., Mao Z., Li B. Non-line-of-sight ultraviolet single-scatter path loss model. - Phonon Network Communications, Oct 05, 2017. - DOI 10.1007/s11107-017-0737-5.

[12]Gagliardi R. M. and Karp S. Optical Communications (New York: Wiley), 1995. - Chap. 6.

[13]Elganimi T.Y. Performance Comparison between OOK, PPM and PAM Modulation Schemes for Free Space Optical (FSO) Communication Systems: Analytical Study. International Journal of Computer Applications 79(11):22-27, October 2013.

[14]Chen G., F. Abou-Galala, Z. Xu, and B. M. Sadler, "Experimental evaluation of LED-based solar blind NLOS communication links," Optics Express, vol. 16, no. 19, pp. 15059-15068, Sep. 2008.

[15]Xu C. and Zhang H., "Packet error rate analysis of IM/DD systems for ultraviolet scattering communications", IEEE Military Communications Conference (2015), pp. 1188-1193.

[16]Ghassemlooy Z., Kaluarachchi E. D., Reyher R. U., and Simmonds A. J., "A new modulation technique based on digital pulse interval modulation (DPIM) for optical-fiber communication,” Microw. Opt. Technol. Lett., vol. 10, no. 1, pp. 1-4, Sep. 1995.

[17]Ghassemlooy Z., and Hayes A. R., "Digital pulse interval modulation for optical communications," IEEE Commun. Mag., vol. 36, no. 12, pp. 95-99, Dec. 1998.

[18]Hu Z., Tang J., "Performance of digital pulse interval modulation of atmospheric optical wireless communication system," Proc. SPIE, vol. 5625, pp. 202-208, Feb. 2005.

[19]Aldibbiat N. M., Ghassemlooy Z., and McLaughlin R., "Error performance of dual header pulse interval modulation (DH-PIM) in optical wireless communications," IEE Proc. Optoelectron., vol. 148, no. 2, pp. 91-96, Apr. 2001

[20]Ma J., Jiang Y., Yu S., Tan L., and Du W., "Packet error rate analysis of OOK, DPIM and PPM modulation schemes for ground-to-satellite optical communications," Opt. Commun., vol. 283, pp. 237-242, Jan. 2010.

[21]Drost R. J., Moore T. J. and Sadler B. M. UV communications channel modeling incorporating multiple scattering interactions, J. Opt. Soc. Am.A28686-95, 2011.

[22]Konstantinov I.S., Vasilyev G.S., Kuzichkin O.R., Surzhik D.I., Lazarev S.A.. Numerical and Analytical Modeling of Wireless UV Communication Channels for the Organization of Wireless Ad-Hoc Network // IJCSNS - International Journal of Computer Science and Network Security - 2018. - Vol. 18, No. 8, pp. 98-104. 